\title{
Numerical Solution of Fifth Order Boundary Value Problems by Petrov-Galerkin Method with Quartic B- Splines as Basis Functions and Quintic B-Splines as Weight Functions
}

\author{
K. N. S. Kasi Viswanadham \\ Department of Mathematics \\ National Institute of Technology \\ Warangal - 506004 India
}

\author{
S. V. Kiranmayi Ch. \\ Department of Mathematics \\ National Institute of Technology \\ Warangal - 506004 India
}

\begin{abstract}
In this paper an efficient numerical scheme to approximate the solutions of fifth-order boundary value problems in a finite domain with two different types of boundary conditions has been prsented, by taking basis functions with quartic Bsplines and weight functions with quintic B-splines in PetrovGalerkin method. In this method, the quartic B-splines and quintic B-splines are redefined into new sets of functions which contain the equal number of functions. The analysis is accompanied by numerical examples. The obtained results demonstrate the reliability and efficiency of the proposed scheme.
\end{abstract}

\section{Keywords}

Basis functions, Boundary value problem, B-splines, PetrovGalerkin method, Weight functions.

\section{INTRODUCTION}

Consider a general linear boundary value problem of fifth order

$$
\begin{aligned}
& p_{0}(t) v^{(5)}+p_{1}(t) v^{(4)}(t)+p_{2}(t) v^{\prime \prime \prime}(t)+p_{3}(t) v^{\prime \prime}(t) \\
& +p_{4}(t) v^{\prime}(t)+p_{5}(t) v(t)=q(t), \quad a<t<b
\end{aligned}
$$

subject to the boundary conditions

$$
\begin{aligned}
& v(a)=A_{0}, v(b)=C_{0}, v^{\prime}(a)=A_{1}, v^{\prime}(b)=C_{1}, v^{\prime \prime}(a)=A_{2}(2 \mathrm{a}) \\
& v(a)=A_{0}, v(b)=C_{0}, \quad(\text { or }) \\
& v^{\prime}(a)+\sigma_{1} v(a)=A_{1}, v^{\prime}(b)+\sigma_{2} v(b)=C_{1}, v^{\prime \prime}(a)=A_{2}
\end{aligned}
$$

where $A_{0}, C_{0}, A_{1}, C_{1}, A_{2}, \sigma_{1}$ and $\sigma_{2}$ are real constants and $\mathrm{p}_{0}(\mathrm{t}), \mathrm{p}_{1}(\mathrm{t}), \mathrm{p}_{2}(\mathrm{t}), \mathrm{p}_{3}(\mathrm{t}), \mathrm{p}_{4}(\mathrm{t}), \mathrm{p}_{5}(\mathrm{t})$ and $\mathrm{q}(\mathrm{t})$ are continuous functions defined in $[a, b]$.

Fifth order boundary value problems generally arise in the mathematical modelling of viscoelastic flows $\left[\begin{array}{ll}1, & 2\end{array}\right]$. Theorems which list the conditions for the existence and uniqueness of solutions of such problems are contained in a comprehensive survey in a book by Agarwal [3],though no numerical methods are contained therein for solving boundary value problems of higher order. Wazwaz [4] developed the solution of special type of fifth order boundary value problems by using the modified Adomain decomposition method and he provided the solution in the form of a rapidly convergent series. Siddiqi et al. [5] presented the solution of a special case of linear fifth order boundary value problems by using quartic spline functions. Rashidinia et al. [6] presented the solution of a special case of linear fifth order boundary value problem by using non-polynomial spline functions technique. Noor and Sayed [7] applied the Homotopy perturbation method for solving fifth order boundary value problems. Caglar and Caglar [8] presented the Local polynomial regression method to solve the special case of fifth order boundary value problems. Gamel [9] presented the solution of fifth order boundary value problems by SincGalerkin method. Syam and Ahili [10] presented combination of Adomain decomposition method and the Homotopy method to solve a fifth order singularly perturbed boundary value problem arising in viscoelastic flows. Zhao [11] developed the solution of fifth order boundary value problems by variational iteration method, Lamnii et al. [12] developed the sextic spline collocation method to solve special case of fifth order boundary value problems. Kasi Viswanadham and Sreenivasulu [13] developed the quartic B-spline Galerkin method to a general fifth order boundary value problem. Petrov Galerkin method with cubic B-splines as basis functions and Quintic B-splines as weight functions [14] have been used to solve a general $5^{\text {th }}$ order boundary value problem. So far, fifth order boundary value problems have not been solved by using Petrov-Galerkin method with quartic Bsplines as basis functions and quintic B-splines as weight functions. This motivated us to solve a fifth order boundary value problem by Petrov-Galerkin method with quartic Bsplines as basis functions and quintic B-splines as weight functions. The present work is motivated by the desire to obtain numerical solutions to higher order boundary value problems with a better accuracy.

Section 2 deals with the justification of using Petrov-Galerkin method. A description of the proposed method with the types of boundary conditions (2) is dealt in section 3 . The procedure of solving the nodal parameters is described in section 4 . The application of the proposed method on solving several examples of linear and nonlinear boundary value problems has been presented in section 5. By using quasilinearization technique [15], a nonlinear problem can be converted into a sequence of linear problems and the limit of solutions of these generated linear problems is the solution of the nonlinear problem. The conclusions are presented in the last section.

\section{JUSTIFICATION OF USING PROPOSED METHOD}

The approximate solution in Finite Element Method (FEM) can be obtained as a linear combination of basis functions 
which constitute a basis for the approximation space under consideration. Petrov-Galerkin method is one of the variational methods involved in FEM. The residual of approximation is made orthogonal to the weight functions in Petrov-Galerkin method. Regardless of properties of the differential operator defined in the given differential equation, a weak form of approximation solution for the differential equation exists and is unique under appropriate conditions $[16,17]$. Further, if we pay sufficient attention to the boundary conditions [18], the weak solution tends to an exact solution of the differential equation. This means that the basis functions should become zero on the boundary where the essential (Dirichlet) type boundary conditions are defined. Also in the Petrov-Galerkin method, the basis functions and the weight functions are equal in number.

\section{DESCRIPTION OF THE PROPOSED METHOD}

\subsection{Quartic B-splines and quintic B-splines:}

The quartic B-splines and quintic B-splines are described in [19-21]. Space variable domain [a, b] is divided into spaced knots (which need not be spaced evenly) given by the partition $a=t_{0}<t_{1}<\ldots<t_{n-1}<t_{n}=b$. Eight additional knots $t_{-4}, t_{-3}, t_{-2}, t_{-1}, t_{\mathrm{n}+1}, t_{\mathrm{n}+2}, t_{n+3}$ and $t_{\mathrm{n}+4}$ are introduced which satisfy the relation

$t_{-4}<t_{-3}<t_{-2}<t_{-1}<t_{0}$ and $t_{\mathrm{n}}<t_{\mathrm{n}+1}<t_{\mathrm{n}+2}<t_{\mathrm{n}+3}<t_{\mathrm{n}+4}$.

Now the quartic B-splines $S_{i}(t)^{\prime} s$ are defined by

$$
S_{i}(t)=\left\{\begin{array}{lr}
\sum_{r=i-2}^{i+3} \frac{\left(t_{r}-t\right)_{+}^{4}}{\pi^{\prime}\left(t_{r}\right)}, & t \in\left[t_{i-2}, t_{i+3}\right] \\
0, & \text { otherwise }
\end{array}\right.
$$

where $\quad\left(t_{r}-t\right)_{+}^{4}= \begin{cases}\left(t_{r}-t\right)^{4}, & \text { if } t_{r} \geq t \\ 0, & \text { if } t_{r} \leq t\end{cases}$

and $\pi(t)=\prod_{r=i-2}^{i+3}\left(t-t_{r}\right)$

where $\left\{S_{-2}(t), S_{-1}(t), S_{0}(t), S_{I}(t), \ldots, S_{n-1}(t), S_{n}(t), S_{n+1}(t)\right\}$ forms a basis for the space $S_{4}(\pi)$ of quartic polynomial splines.

Schoenberg [21] has shown that quartic B-splines are the unique nonzero splines of smallest compact support with the knots at $t_{-4}<t_{-3}<t_{-2}<t_{-1}<t_{0}<t_{1}<\ldots<t_{\mathrm{n}-1}<t_{\mathrm{n}}<t_{\mathrm{n}+1}<t_{\mathrm{n}+2}<t_{\mathrm{n}+3}<t_{\mathrm{n}+4}$.

In the same way, the quintic B-splines $R_{\mathrm{i}}(t)$ 's are defined by

$$
R_{i}(t)=\left\{\begin{array}{lc}
\sum_{r=i-3}^{i+3} \frac{\left(t_{r}-t\right)_{+}^{5}}{\pi^{\prime}\left(t_{r}\right)}, & x \in\left[t_{i-3}, t_{i+3}\right] \\
0, & \text { otherwise }
\end{array}\right.
$$

where $\quad\left(t_{r}-t\right)_{+}^{5}= \begin{cases}\left(t_{r}-t\right)^{5}, & \text { if } t_{r} \geq t \\ 0, & \text { if } t_{r} \leq t\end{cases}$

and $\quad \pi(t)=\prod_{r=i-3}^{i+3}\left(t-t_{r}\right)$ where $\quad\left\{R_{-2}(t), R_{-1}(t), R_{0}(t), R_{l}(t), \ldots, R_{n-1}(t), R_{n}(t), R_{n+1}(t), R_{n+2}(t)\right\}$ forms a basis for the space $S_{5}(\pi)$ of quintic polynomial splines by introducing two more additional knots $t_{-5}, t_{n+5}$ to the already existing knots $t_{-4}$ to $t_{n+4}$. Schoenberg [21] has shown that quintic B-splines are the unique nonzero splines of smallest compact support with the knots at

$t_{-5}<t_{-4}<t_{-3}<t_{-2}<t_{-1}<t_{0}<t_{1}<\ldots<t_{\mathrm{n}-1}<t_{\mathrm{n}}<t_{\mathrm{n}+1}<t_{\mathrm{n}+2}<t_{\mathrm{n}+3}<t_{\mathrm{n}+4}<t_{n+5}$.

The approximation for $v(t)$ is defined as

$$
v(t)=\sum_{j=-2}^{n+1} \alpha_{j} S_{j}(t)
$$

where $\alpha_{j} s$ are the nodal parameters to be determined and $S_{j}(t)^{\prime} s$ are the quartic B-spline basis functions. In PetrovGalerkin method, the basis functions should be zero on the boundary where the essential type of boundary conditions are prescribed. In the set of quartic B-splines $\left\{S_{-2}(t), S_{-1}(t), S_{0}(t)\right.$, $\left.S_{1}(t), \ldots, S_{n-1}(t), S_{n}(t), S_{n+1}(t)\right\}$, the basis functions $S_{-2}(t), S_{-1}(t)$, $S_{0}(t), S_{I}(t)$ do not become zero on the left boundary and $S_{\mathrm{n}-2}(t)$, $S_{\mathrm{n}-1}(t), S_{\mathrm{n}}(t)$ and $S_{\mathrm{n}+1}(t)$ do not become zero on the right boundary. So, it is necessary to redefine the basis functions into a new set of basis functions which become zero on the boundary where the essential type boundary conditions are specified. When the chosen approximation satisfies the prescribed boundary conditions or most of the boundary conditions, it gives better approximation results. In view of this, the basis functions are redefined into a new set of basis functions which vanish on the boundary where the Dirichlet, the Neumann or mixed boundary condition at the left boundary are prescribed.

\subsection{Redefinition of basis functions with boundary conditions (2a)}

Applying the essential boundary conditions of (2), the approximate solution $v(t)$ at the boundary points can be taken as

$$
\begin{aligned}
& A_{0}=v(a)=v\left(t_{0}\right)=\alpha_{-2} S_{-2}\left(t_{0}\right)+\alpha_{-1} S_{-1}\left(t_{0}\right) \\
& +\alpha_{0} S_{0}\left(t_{0}\right)+\alpha_{1} S_{1}\left(t_{0}\right) \\
& C_{0}=v(b)=v\left(t_{n}\right)=\alpha_{n-2} S_{n-2}\left(t_{n}\right)+\alpha_{n-1} S_{n-1}\left(t_{n}\right) \\
& +\alpha_{n} S_{n}\left(t_{n}\right)+\alpha_{n+1} S_{n+1}\left(t_{n}\right)
\end{aligned}
$$

Eliminating $\alpha_{-2}$ and $\alpha_{n+1}$ from the equations (3), (4) and (5), we get

$$
v(t)=w_{1}(t)+\sum_{j=-1}^{n} \alpha_{j} P_{j}(t)
$$

where

$$
\begin{aligned}
& w_{1}(t)=\frac{A_{0}}{S_{-2}\left(t_{0}\right)} S_{-2}(t)+\frac{C_{0}}{S_{n+1}\left(t_{n}\right)} S_{n+1}(t) \\
& P_{j}(t)=\left\{\begin{array}{lr}
S_{j}(t)-\frac{S_{j}\left(t_{0}\right)}{S_{-2}\left(t_{0}\right)} S_{-2}(t), & j=-1,0,1 \\
S_{j}(t), & j=2,3, \ldots, n-3 \\
S_{j}(t)-\frac{S_{j}\left(t_{n}\right)}{S_{n+1}\left(t_{n}\right)} S_{n+1}(t), & j=n-2, n-1, n
\end{array}\right.
\end{aligned}
$$


Using the left Neumann boundary conditions of (2a) to the approximate solution $v(t)$ in (6), we get

$$
\begin{aligned}
& A_{1}=v^{\prime}(a)=v^{\prime}\left(t_{0}\right)=w_{1}^{\prime}\left(t_{0}\right)+\alpha_{-1} P_{-1}^{\prime}\left(t_{0}\right)+\alpha_{0} P_{0}^{\prime}\left(t_{0}\right) \\
& +\alpha_{1} P_{1}^{\prime}\left(t_{0}\right)
\end{aligned}
$$

Eliminating $\alpha_{-1}$ from the equations (6) and (9), we get the approximation for $v(t)$ as

$$
v(t)=w(t)+\sum_{j=0}^{n} \alpha_{j} \tilde{B}_{j}(t)
$$

where

$$
\begin{aligned}
& w(t)=w_{1}(t)+\frac{A_{1}-w_{1}^{\prime}\left(t_{0}\right)}{P_{-1}^{\prime}\left(t_{0}\right)} P_{-1}(t) \\
& \tilde{B}_{j}(t)= \begin{cases}P_{j}(t)-\frac{P_{j}^{\prime}\left(t_{0}\right)}{P_{-1}^{\prime}\left(t_{0}\right)} P_{-1}(t), & j=0,1 \\
P_{j}(t), & j=2,3, \ldots, n\end{cases}
\end{aligned}
$$

\subsection{Redefinition of basis functions with boundary conditions ( 2 b)}

Using the left mixed boundary conditions of (2b) to the approximate solution $v(t)$ in (6), we get

$$
\begin{aligned}
& A_{1}=v^{\prime}(a)+\sigma_{1} v(a)=v^{\prime}\left(t_{0}\right)+\sigma_{1} v\left(t_{0}\right)=w_{1}^{\prime}\left(t_{0}\right)+\alpha_{-1} P_{-1}^{\prime}\left(t_{0}\right) \\
& +\alpha_{0} P_{0}^{\prime}\left(t_{0}\right)+\alpha_{1} P_{1}^{\prime}\left(t_{0}\right)+\sigma_{1} w_{1}\left(t_{0}\right)
\end{aligned}
$$

Eliminating $\alpha_{-1}$ from the equations (13) and (6), we get approximation for $v(t)$ as

$$
v(t)=w(t)+\sum_{j=0}^{n} \alpha_{j} \tilde{B}_{j}(t)
$$

where

$$
w(t)=w_{1}(t)+\frac{A_{1}-w_{1}^{\prime}\left(t_{0}\right)-\sigma_{1} w_{1}\left(t_{0}\right)}{P_{1}^{\prime}\left(t_{0}\right)} P_{-1}(t)
$$

and $\tilde{B}_{j}(t)$ 's are as defined in (12).

$\left\{\tilde{B}_{j}(t), j=0,1, \ldots, n\right\}$ is the new set of basis functions for the approximation $v(t)$. Here $w(t)$ takes care of given set of essential and left Neumann or mixed type of boundary conditions and $\tilde{B}_{j}(t)$ 's are vanishing at the boundary and their first derivative vanish at left boundary. In the proposed method, the new set of basis functions and weight functions should be equal in number. Here the number of basis functions in the approximation for $v(t)$ in (6) is $\mathrm{n}+1$ and the number of weight functions is $n+5$. So, it is necessary to redefine the weight functions into a new set of weight functions which are equal in number of the basis functions.

Assume that the approximation for $u(t)$ as

$u(t)=\sum_{j=-2}^{n+2} \beta_{j} R_{j}(t)$
3.4 Method with boundary conditions (2a)

Assume that approximation $u(t)$, given by (16), satisfies the conditions

$u(a)=0, u(b)=0, u^{\prime}(a)=0, u^{\prime}(b)=0$

Using (16) and (17), we get the approximate solution for $u(t)$ at the boundary points as

$$
\begin{aligned}
& u(a)=u\left(t_{0}\right)=\sum_{j=-2}^{2} \beta_{j} R_{j}\left(t_{0}\right)=0 \\
& u(b)=u\left(t_{n}\right)=\sum_{j=n-2}^{n+2} \beta_{j} R_{j}\left(t_{n}\right)=0 \\
& u^{\prime}(a)=u^{\prime}\left(t_{0}\right)=\sum_{j=-2}^{2} \beta_{j} R_{j}^{\prime}\left(t_{0}\right)=0 \\
& u^{\prime}(b)=u^{\prime}\left(t_{n}\right)=\sum_{j=n-2}^{n+2} \beta_{j} R_{j}^{\prime}\left(t_{n}\right)=0
\end{aligned}
$$

Eliminating $\beta_{-2}, \beta_{-1}, \beta_{\mathrm{n}+1}$ and $\beta_{\mathrm{n}+2}$ from the equations (16) and (18) to (21), we get the approximation for $u(t)$ as

$$
u(t)=\sum_{j=0}^{n} \beta_{j} T_{j}(t)
$$

where

$$
\begin{aligned}
& T_{j}(t)= \begin{cases}Q_{j}(t)-\frac{Q_{j}^{\prime}\left(t_{0}\right)}{Q_{-1}^{\prime}\left(t_{0}\right)} Q_{-1}(t), & j=0,1,2 \\
Q_{j}(t), & j=3,4, \ldots, n \\
Q_{j}(t)-\frac{Q_{j}^{\prime}\left(t_{n}\right)}{Q_{n+1}^{\prime}\left(t_{n}\right)} Q_{n+1}(t), & j=n-2, n-1, n .\end{cases} \\
& Q_{j}(t)= \begin{cases}R_{j}(t)-\frac{R_{j}\left(t_{0}\right)}{R_{-2}\left(t_{0}\right)} R_{-2}(t), & j=-1,0,1,2 \\
R_{j}(t), & j=3,4, \ldots, n-3 \\
R_{j}(t)-\frac{R_{j}\left(t_{n}\right)}{R_{n+2}\left(t_{n}\right)} R_{n+2}(t), & j=n-2, n-1, n, n+1\end{cases}
\end{aligned}
$$

Now the new set of weight functions for the approximation $u(t)$ is $\left\{T_{j}(t), j=0,1, \ldots, n\right\}$. Here

$$
T_{j}\left(t_{0}\right)=T_{j}\left(t_{n}\right)=T_{j}^{\prime}\left(t_{0}\right)=T_{j}^{\prime}\left(t_{n}\right)=0 \text { for all } \mathrm{j} .
$$

Applying the proposed method to (1) with the new set of basis functions $\left\{\tilde{B}_{j}(t), j=0,1, \ldots, n\right\}$ defined in (12) and with the new set of weight functions $\left\{T_{j}(t), j=0,1, \ldots, n\right\}$ defined in (23), we get

$$
\begin{aligned}
& \int_{t_{0}}^{t_{n}}\left[p_{0}(t) v^{(5)}(t)+p_{1}(t) v^{(4)}(t)+p_{2}(t) v^{\prime \prime \prime}(t)+p_{3}(t) v^{\prime \prime}(t)\right. \\
& \left.+p_{4}(t) v^{\prime}(t)+p_{5}(t) v(t)\right] T_{i}(t) d t=\int_{t_{0}}^{t_{n}} b(t) T_{i}(t) d t
\end{aligned}
$$


for $\mathrm{i}=0,1,2, \ldots, \mathrm{n}$.

Integrating by parts the first two terms on the left hand side of (25) and after applying the boundary conditions mentioned in (2a), we get

$$
\begin{aligned}
& \int_{t_{0}}^{t_{n}} p_{0}(t) T_{i}(t) v^{(5)}(t) d t=\frac{d^{2}}{d t^{2}}\left[p_{0}(t) T_{i}(t)\right]_{t_{n}} v^{\prime \prime}\left(t_{n}\right) \\
& -\frac{d^{2}}{d t^{2}}\left[p_{0}(t) T_{i}(t)\right]_{t_{0}} A_{2}-\frac{d^{3}}{d t^{3}}\left[p_{0}(t) T_{i}(t)\right]_{t_{n}} C_{1} \\
& +\frac{d^{3}}{d t^{3}}\left[p_{0}(t) T_{i}(t)\right]_{t_{0}} A_{1}+\int_{t_{0}}^{t_{n}} \frac{d^{4}}{d t^{4}}\left[p_{0}(t) T_{i}(t)\right] v^{\prime}(t) d t \\
& \int_{t_{0}}^{t_{n}} p_{1}(t) T_{i}(t) v^{(4)}(t) d t=\int_{t_{0}}^{t_{n}} \frac{d^{2}}{d t^{2}}\left[p_{1}(t) T_{i}(t)\right] v^{\prime \prime}(t) d t
\end{aligned}
$$

Using (26), (27) and (10) in (25) and after rearrangement, we get a system of equations in the matrix form as

$$
\boldsymbol{K} \boldsymbol{\alpha}=\boldsymbol{f}
$$

where $\quad \boldsymbol{K}=\left[k_{i j}\right]$;

$$
\begin{aligned}
& k_{i j}=\int_{t_{0}}^{t_{n}}\left\{\left[\frac{d^{4}}{d t^{4}}\left[p_{0}(t) T_{i}(t)\right]+P_{4}(t) T_{i}(t)\right] \tilde{B}_{j}^{\prime}(t)\right. \\
& +\left[\frac{d^{2}}{d t^{2}}\left[p_{1}(t) T_{i}(t)\right]+p_{3}(t) T_{i}(t)\right] \tilde{B}_{j}^{\prime \prime}(t) \\
& \left.+p_{2}(t) T_{i}(t) \tilde{B}_{j}^{\prime \prime \prime}(t)+p_{5}(t) T_{i}(t) \tilde{B}_{j}(t)\right\} d t \\
& +\frac{d^{2}}{d t^{2}}\left[p_{0}(t) T_{i}(t)\right]_{t_{n}} \tilde{B}_{j}^{\prime \prime}\left(t_{n}\right) \\
& \text { for } \mathrm{i}=0,1,2, \ldots, \mathrm{n} ; \mathrm{j}=0,1,2, \ldots, \mathrm{n}
\end{aligned}
$$

$\boldsymbol{f}=\left[f_{i}\right]$

$f_{i}=\int_{t_{0}}^{t_{n}}\left\{q(t) T_{i}(t)-\left[\frac{d^{4}}{d t^{4}}\left[p_{0}(t) T_{i}(t)\right]+P_{4}(t) T_{i}(t)\right] w^{\prime}(t)\right.$

$-\left[\frac{d^{2}}{d t^{2}}\left[p_{1}(t) T_{i}(t)\right]+p_{3}(t) T_{i}(t)\right] w^{\prime \prime}(t)$

$\left.-p_{2}(t) T_{i}(t) w^{\prime \prime \prime}(t)-p_{5}(t) T_{i}(t) w(t)\right\} d t$

$-\frac{d^{2}}{d t^{2}}\left[p_{0}(t) T_{i}(t)\right]_{t_{n}} w^{\prime \prime}\left(t_{n}\right)+\frac{d^{2}}{d t^{2}}\left[p_{0}(t) T_{i}(t)\right]_{t_{0}} A_{2}$

$+\frac{d^{3}}{d t^{3}}\left[p_{0}(t) T_{i}(t)\right]_{t_{n}} C_{1}-\frac{d^{3}}{d t^{3}}\left[p_{0}(t) T_{i}(t)\right]_{t_{0}} A_{1}$

$$
\text { for } \mathrm{i}=0,1,2, \ldots, \mathrm{n}
$$

and

$$
\alpha=\left[\alpha_{0} \alpha_{1} \ldots \alpha_{n}\right]^{T}
$$

\subsection{Method with boundary conditions (2b)}

Here also assume that approximation $u(t)$, given by (16), satisfies the conditions defined in (17).

Proceeding as in section 3.4, we get $\left\{T_{j}(t), j=0,1, \ldots, n\right\}$ defined in (23), are the weight functions for the approximation $u(t)$.
Applying the proposed method to (1) with the set of basis functions $\left\{\tilde{B}_{j}(t), j=0,1, \ldots, n\right\}$ defined in (12) and with the set of weight functions $\left\{T_{j}(t), j=0,1, \ldots, n\right\}$ defined in (23), we get

$$
\begin{aligned}
& \int_{t_{0}}^{t_{n}}\left[p_{0}(t) v^{(5)}(t)+p_{1}(t) v^{(4)}(t)+p_{2}(t) v^{\prime \prime \prime}(t)+p_{3}(t) v^{\prime \prime}(t)\right. \\
& \left.+p_{4}(t) v^{\prime}(t)+p_{5}(t) v(t)\right] T_{i}(t) d t=\int_{t_{0}}^{t_{n}} b(t) T_{i}(t) d t
\end{aligned}
$$

$$
\text { for } \mathrm{i}=0,1,2, \ldots, \mathrm{n}
$$

Integrating by parts the first two terms on the left hand side of (31) and after applying the boundary conditions mentioned in (2b), we get

$$
\begin{aligned}
& \int_{t_{0}}^{t_{n}} p_{0}(t) T_{i}(t) v^{(5)}(t) d t=\frac{d^{2}}{d t^{2}}\left[p_{0}(t) T_{i}(t)\right]_{t_{n}} v^{\prime \prime}\left(t_{n}\right) \\
& -\frac{d^{2}}{d t^{2}}\left[p_{0}(t) T_{i}(t)\right]_{t_{0}} A_{2}-\frac{d^{3}}{d t^{3}}\left[p_{0}(t) T_{i}(t)\right]_{t_{n}}\left(C_{1}-\sigma_{2} C_{0}\right) \\
& +\frac{d^{3}}{d t^{3}}\left[p_{0}(t) T_{i}(t)\right]_{t_{0}}\left(A_{1}-\sigma_{1} A_{0}\right) \\
& +\int_{t_{0}}^{t_{n}} \frac{d^{4}}{d t^{4}}\left[p_{0}(t) T_{i}(t)\right] v^{\prime}(t) d t \\
& \int_{t_{0}}^{t_{n}} p_{1}(t) T_{i}(t) v^{(4)}(t) d t=\int_{t_{0}}^{t_{n}} \frac{d^{2}}{d t^{2}}\left[p_{1}(t) T_{i}(t)\right] v^{\prime \prime}(t) d t
\end{aligned}
$$

Using (32), (33) and (14) in (31) and after rearrangement, we get a system of equations in the matrix form as

$$
\boldsymbol{K} \boldsymbol{\alpha}=\boldsymbol{f}
$$

where $\quad \boldsymbol{K}=\left[k_{i j}\right]$;

$$
\begin{aligned}
& k_{i j}=\int_{t_{0}}^{t_{n}}\left\{\left[\frac{d^{4}}{d t^{4}}\left[p_{0}(t) T_{i}(t)\right]+P_{4}(t) T_{i}(t)\right] \tilde{B}_{j}^{\prime}(t)\right. \\
& +\left[\frac{d^{2}}{d t^{2}}\left[p_{1}(t) T_{i}(t)\right]+p_{3}(t) T_{i}(t)\right] \tilde{B}_{j}^{\prime \prime}(t) \\
& \left.+p_{2}(t) T_{i}(t) \tilde{B}_{j}^{\prime \prime \prime}(t)+p_{5}(t) T_{i}(t) \tilde{B}_{j}(t)\right\} d t \\
& +\frac{d^{2}}{d t^{2}}\left[p_{0}(t) T_{i}(t)\right]_{t_{n}} \tilde{B}_{j}^{\prime \prime}\left(t_{n}\right) \\
& \text { for } \mathrm{i}=0,1,2, \ldots, \mathrm{n} ; \mathrm{j}=0,1,2, \ldots, \mathrm{n}
\end{aligned}
$$




$$
\begin{aligned}
& f_{i}=\int_{t_{0}}^{t_{n}}\left\{q(t) T_{i}(t)-\left[\frac{d^{4}}{d t^{4}}\left[p_{0}(t) T_{i}(t)\right]+P_{4}(t) T_{i}(t)\right] w^{\prime}(t)\right. \\
& -\left[\frac{d^{2}}{d t^{2}}\left[p_{1}(t) T_{i}(t)\right]+p_{3}(t) T_{i}(t)\right] w^{\prime \prime}(t) \\
& \left.-p_{2}(t) T_{i}(t) w^{\prime \prime \prime}(t)-p_{5}(t) T_{i}(t) w(t)\right\} d t \\
& -\frac{d^{2}}{d t^{2}}\left[p_{0}(t) T_{i}(t)\right]_{t_{n}} w^{\prime \prime}\left(t_{n}\right)+\frac{d^{2}}{d t^{2}}\left[p_{0}(t) T_{i}(t)\right]_{t_{0}} A_{2} \\
& +\frac{d^{3}}{d t^{3}}\left[p_{0}(t) T_{i}(t)\right]_{t_{n}}\left(C_{1}-\sigma_{2} C_{0}\right)-\frac{d^{3}}{d t^{3}}\left[p_{0}(t) T_{i}(t)\right]_{t_{0}}\left(A_{1}-\sigma_{1} A_{0}\right) \\
& \text { and } \quad \text { for } \quad \mathrm{i}=0,1,2, \ldots, \mathrm{n} \\
& \qquad \alpha=\left[\alpha_{0} \alpha_{1} \ldots \alpha_{n}\right]^{T} .
\end{aligned}
$$

\section{PROCEDURE OF SOLVING THE NODAL PARAMETERS}

A general element in the matrix $\boldsymbol{K}$ is given by $\sum_{m=0}^{n-1} I_{m}$, where $I_{m}=\int_{t_{m}}^{t_{m+1}} u_{i}(t) r_{j}(t) M(t) d t, r_{j}(t)$ are the quartic B-spline basis functions or their derivatives and $u_{i}(t)$ are the quintic B-spline weight functions or their derivatives. Here $I_{m}=0$ if $\left(t_{j-2}, t_{j+3}\right) \cap\left(t_{i-3}, t_{i+3}\right) \cap\left(t_{m}, t_{m+1}\right)=\varnothing$. For the evaluation of each $I_{m}$, we have used 5-point Gauss-Legendre quadrature formula. Due to this, the stiffness matrix $\boldsymbol{K}$ is a ten diagonal band matrix. Solving the system $\boldsymbol{K} \boldsymbol{\alpha}=\boldsymbol{f}$ by using the band matrix solution package, we get the nodal parameter vector $\boldsymbol{\alpha}$. We have used the FORTRAN-90 code to solve the boundary value problems (1) - (2) by the proposed method.

\section{NUMERICAL EXAMPLES}

To test the accuracy and efficiency of the developed method, we solved five linear and two nonlinear fifth order boundary value problems. The obtained numerical results for each problem are presented in tabular forms.

Example 1: Consider the following linear boundary value problem

$$
v^{(5)}-v=-(15+10 t) e^{t}, \quad 0 \leq t \leq 1
$$

subject to $\quad v(0)=v(1)=0, v^{\prime}(0)=1$,

$v^{\prime}(1)=-e, v^{\prime \prime}(0)=0$.

The exact solution for the above problem is

$v=t e^{t}(1-t)$.

Dividing the domain $[0,1]$ into 10 equal subintervals, the numerical results obtained for this problem are presented in Table 1.

Table 1: Numerical results for Example 1

\begin{tabular}{|c|c|}
\hline$t$ & $\begin{array}{c}\text { Absolute error by } \\
\text { proposed method }\end{array}$ \\
\hline 0.1 & $1.788139 \mathrm{E}-07$ \\
0.2 & $5.811453 \mathrm{E}-07$ \\
0.3 & $4.172325 \mathrm{E}-07$ \\
\hline
\end{tabular}

\begin{tabular}{|l|l|}
\hline 0.4 & $1.341105 \mathrm{E}-06$ \\
0.5 & $2.413988 \mathrm{E}-06$ \\
0.6 & $3.010035 \mathrm{E}-06$ \\
0.7 & $2.324581 \mathrm{E}-06$ \\
0.8 & $1.400709 \mathrm{E}-06$ \\
0.9 & $4.917383 \mathrm{E}-07$ \\
\hline
\end{tabular}

Example 2: Consider the following linear boundary value problem

$$
\begin{aligned}
& v^{(5)}+t v=19 t \cos t+2 t^{3} \cos t+41 \sin t \\
& -2 t^{2} \sin t, \quad-1<t<1
\end{aligned}
$$

subject to $\quad v(-1)=v(1)=\cos 1$,

$v^{\prime}(-1)=-v^{\prime}(1)=-4 \cos 1+\sin 1, \quad v^{\prime \prime}(-1)=3 \cos 1-8 \sin 1$.

The exact solution for the above problem is

$v=\left(2 t^{2}-1\right) \cos t$.

Dividing the domain $[-1,1]$ into 10 equal subintervals, the numerical results obtained for this problem are presented in Table 2.

Table 2: Numerical results for Example 2

\begin{tabular}{|c|c|}
\hline$t$ & $\begin{array}{c}\text { Absolute error by } \\
\text { proposed method }\end{array}$ \\
\hline-0.8 & $1.132488 \mathrm{E}-06$ \\
-0.6 & $4.470348 \mathrm{E}-08$ \\
-0.4 & $7.450581 \mathrm{E}-06$ \\
-0.2 & $1.561642 \mathrm{E}-05$ \\
0.0 & $2.050400 \mathrm{E}-05$ \\
0.2 & $2.127886 \mathrm{E}-05$ \\
0.4 & $1.770258 \mathrm{E}-05$ \\
0.6 & $9.968877 \mathrm{E}-06$ \\
0.8 & $2.905726 \mathrm{E}-06$ \\
\hline
\end{tabular}

Example 3: Consider the following linear boundary value problem

$v^{(5)}-v^{(4)}=-e^{t}(2 t+7), \quad 0<t<1$

subject to

$$
\begin{aligned}
& v(0)=0, v(1)=0, v^{\prime}(0)-v(0)=1, \\
& v^{\prime}(1)-v(1)=-e, v^{\prime \prime}(0)=0 .
\end{aligned}
$$

The exact solution for the above problem is $v=t e^{t}(1-t)$.

Dividing the domain $[0,1]$ into 10 equal subintervals, the numerical results obtained for this problem are presented in Table 3.

Table 3: Numerical results for Example 3

\begin{tabular}{|c|c|}
\hline$t$ & $\begin{array}{c}\text { Absolute error by } \\
\text { proposed method }\end{array}$ \\
\hline 0.1 & $2.011657 \mathrm{E}-07$ \\
0.2 & $6.854534 \mathrm{E}-07$ \\
0.3 & $6.854534 \mathrm{E}-07$ \\
0.4 & $1.758337 \mathrm{E}-06$ \\
0.5 & $2.950430 \mathrm{E}-06$ \\
\hline
\end{tabular}




\begin{tabular}{|l|l|}
\hline 0.6 & $3.486872 \mathrm{E}-06$ \\
0.7 & $2.592802 \mathrm{E}-06$ \\
0.8 & $1.460314 \mathrm{E}-06$ \\
0.9 & $5.066395 \mathrm{E}-07$ \\
\hline
\end{tabular}

Example 4: Consider the following linear boundary value problem

$$
\begin{aligned}
v^{(5)}+ & v^{(4)}+e^{-2 t} v=e^{-t}\left[-4 e^{2 t}(-3+t) \cos t\right. \\
& \left.-\left\{1-t+4 e^{2 t}(5+2 t) \sin t\right\}\right], \quad 0<t<1
\end{aligned}
$$

subject to

$$
\begin{aligned}
& v(0)=0, v(1)=0, v^{\prime}(0)=-1, \\
& v^{\prime}(1)=e \sin 1, v^{\prime \prime}(0)=0 .
\end{aligned}
$$

The exact solution for the above problem is

$$
v=e^{t}(t-1) \sin t \text {. }
$$

Dividing the domain $[0,1]$ into 10 equal subintervals, the numerical results obtained for this problem are presented in Table 4.

\section{Table 4: Numerical results for Example 4}

\begin{tabular}{|c|c|}
\hline$t$ & $\begin{array}{c}\text { Absolute error by } \\
\text { proposed method }\end{array}$ \\
\hline 0.1 & $1.639128 \mathrm{E}-07$ \\
0.2 & $3.725290 \mathrm{E}-07$ \\
0.3 & $2.980232 \mathrm{E}-08$ \\
0.4 & $3.576279 \mathrm{E}-07$ \\
0.5 & $9.536743 \mathrm{E}-07$ \\
0.6 & $1.221895 \mathrm{E}-06$ \\
0.7 & $7.152557 \mathrm{E}-07$ \\
0.8 & $2.384186 \mathrm{E}-07$ \\
0.9 & $2.235174 \mathrm{E}-07$ \\
\hline
\end{tabular}

Example 5: Consider the following linear boundary value problem

$$
\begin{aligned}
& v^{(5)}+(t-2) v^{(4)}+2 v^{\prime \prime \prime}-\left(t^{2}+2 t-1\right) v^{\prime \prime} \\
& +\left(2 t^{2}+4 t\right) v^{\prime}-2 t^{2} v=4 e^{t} \cos t-2 t^{4}+4 t^{3} \\
& +6 t^{2}-4 t+2, \quad 0<t<1
\end{aligned}
$$

subject to

$$
\begin{aligned}
& v(0)=0, \quad v(1)=1+2 e \sin 1, v^{\prime}(0)=2, \\
& v^{\prime}(1)=2 e(\cos 1+\sin 1)+2, v^{\prime \prime}(0)=6 .
\end{aligned}
$$

The exact solution for the above problem is

$$
v=t^{2}+2 e^{t} \sin t .
$$

Dividing the domain $[0,1]$ into 10 equal subintervals, the numerical results obtained for this problem are presented in Table 5.

Table 5: Numerical results for Example 5

\begin{tabular}{|c|c|}
\hline$t$ & $\begin{array}{c}\text { Absolute error by } \\
\text { proposed method }\end{array}$ \\
\hline 0.1 & $4.768372 \mathrm{E}-07$ \\
0.2 & $6.556511 \mathrm{E}-07$ \\
\hline
\end{tabular}

\begin{tabular}{|l|l|}
\hline 0.3 & $2.384186 \mathrm{E}-06$ \\
0.4 & $2.264977 \mathrm{E}-06$ \\
0.5 & $1.192093 \mathrm{E}-06$ \\
0.6 & $5.960464 \mathrm{E}-06$ \\
0.7 & $5.245209 \mathrm{E}-06$ \\
0.8 & $3.576279 \mathrm{E}-06$ \\
0.9 & $7.152557 \mathrm{E}-06$ \\
\hline
\end{tabular}

Example 6: Consider the following nonlinear boundary value problem

$v^{(5)}+\left(v^{\prime}\right)^{2} e^{4 v}-4 v^{2} e^{v^{\prime \prime}}+e^{2 t}\left(v^{\prime \prime \prime}\right)^{2}$

$=32 e^{-2 t}, \quad 0<t<1$

subject to

$v(0)=1, v(1)=\mathrm{e}^{-2}, v^{\prime}(0)-v(0)=-3$,

$v^{\prime}(1)-v(1)=-3 e^{-2}, v^{\prime \prime}(0)=4$.

The exact solution for the above problem is $v=e^{-2 t}$.

By using quasilinearization technique [15], the nonlinear boundary value problem (42) is converted into a sequence of linear boundary value problems as

$$
\begin{gathered}
v_{(n+1)}^{(5)}+\left[2 e^{2 t} v_{(n)}^{\prime \prime \prime}\right] v_{(n+1)}^{\prime \prime \prime}-4\left[v_{(n)}\right]^{2} e^{v_{(n)}^{\prime \prime}} v_{(n+1)}^{\prime \prime} \\
+2 v_{(n)}^{\prime} e^{4 v_{(n)}} v_{(n+1)}^{\prime}+\left[4\left[v_{(n)}^{\prime}\right]^{2} e^{4 v_{(n)}}-8 v_{(n)} e^{v_{(n)}^{\prime \prime}}\right] v_{(n+1)} \\
=e^{2 t}\left[v_{(n)}^{\prime \prime \prime}\right]^{2}+4 v_{(n)}^{2} e^{v_{(n)}^{\prime \prime}}\left(1-v_{(n)}^{\prime \prime}\right)+\left[v_{(n)}^{\prime}\right]^{2} e^{4 v_{(n)}} \\
+32 e^{-2 t}+\left(4\left[v_{(n)}^{\prime}\right]^{2} e^{4 v_{(n)}}-8 v_{(n)} e^{v_{(n)}^{\prime \prime}}\right) v_{(n)}, \\
\mathrm{n}=0,1,2, \ldots
\end{gathered}
$$

subject to

$$
\begin{gathered}
v_{(n+1)}(0)=1, v_{(n+1)}(1)=e^{-2}, v_{(n+1)}^{\prime}(0)-v_{(n+1)}(0)=-3, \\
v_{(n+1)}^{\prime}(1)-v_{(n+1)}(1)=-3 e^{-2}, v_{(n+1)}^{\prime \prime}(0)=4 .
\end{gathered}
$$

Here $v_{(n+1)}$ is the $(n+1)^{\text {th }}$ approximation for $v$. The domain

$[0,1]$ is divided into 10 equal subintervals and the proposed method is applied to the sequence of linear problems (43). The obtained numerical results for this problem are presented in Table 6 .

Table 6: Numerical results for Example 6

\begin{tabular}{|c|c|}
\hline$t$ & $\begin{array}{c}\text { Absolute error by } \\
\text { proposed method }\end{array}$ \\
\hline 0.1 & $1.966953 \mathrm{E}-06$ \\
0.2 & $1.072884 \mathrm{E}-05$ \\
0.3 & $2.568960 \mathrm{E}-05$ \\
0.4 & $4.595518 \mathrm{E}-05$ \\
0.5 & $6.499887 \mathrm{E}-05$ \\
0.6 & $7.620454 \mathrm{E}-05$ \\
0.7 & $7.419288 \mathrm{E}-05$ \\
0.8 & $5.659461 \mathrm{E}-05$ \\
0.9 & $2.484024 \mathrm{E}-05$ \\
\hline
\end{tabular}

Example 7: Consider the following nonlinear boundary value problem

$v^{(5)}+v^{(4)}+e^{-2 t} v^{2}=2 e^{t}+1, \quad 0<t<1$ 
subject to

$v(0)=1, v(1)=e, v^{\prime}(0)=1, v^{\prime}(1)=e, v^{\prime \prime}(0)=1$.

The exact solution for the above problem is $v=e^{t}$.

By using quasilinearization technique [15], the nonlinear boundary value problem (44) is converted into a sequence of linear boundary value problems as

$v_{(n+1)}^{(5)}+v_{(n+1)}^{(4)}+2 e^{-2 t} v_{(n)} v_{(n+1)}$

$=2 e^{t}+e^{-2 t} v_{(n)}^{2}+1, \quad n=0,1,2, \ldots$

subject to

$$
\begin{aligned}
& v_{(n+1)}(0)=1, v_{(n+1)}(1)=e, v_{(n+1)}^{\prime}(0)=1, \\
& v_{(n+1)}^{\prime}(1)=e, v_{(n+1)}^{\prime \prime}(0)=1 .
\end{aligned}
$$

Here $v_{(n+1)}$ is the $(n+1)^{\text {th }}$ approximation for $v$. The domain $[0,1]$ is divided into 10 equal subintervals and the proposed method is applied to the sequence of linear problems (45). The obtained numerical results for this problem are presented in Table 7.

Table 7: Numerical results for Example 7

\begin{tabular}{|c|c|}
\hline$t$ & $\begin{array}{c}\text { Absolute error by } \\
\text { proposed method }\end{array}$ \\
\hline 0.1 & $1.072884 \mathrm{E}-06$ \\
0.2 & $3.814697 \mathrm{E}-06$ \\
0.3 & $3.814697 \mathrm{E}-06$ \\
0.4 & $7.748604 \mathrm{E}-06$ \\
0.5 & $1.323223 \mathrm{E}-05$ \\
0.6 & $1.680851 \mathrm{E}-05$ \\
0.7 & $1.430511 \mathrm{E}-05$ \\
0.8 & $8.821487 \mathrm{E}-06$ \\
0.9 & $4.053116 \mathrm{E}-06$ \\
\hline
\end{tabular}

\section{CONCLUSIONS}

In this paper, we have solved a general fifth order two point boundary value problem with two different cases of boundary conditions by the proposed method with quartic B-splines as basis functions and quintic B-splines as weight functions. The quartic B-splines and quintic B-splines are redefined into new sets of functions which contain the equal number of functions. To test the accuracy and efficiency of the developed method, it has been tested on five linear and two nonlinear fifth order boundary value problems. It is found that the obtained results are giving a little error. The strength of the developed method lies in the easiness of its application, accuracy and efficiency.

\section{REFERENCES}

[1] A.R.Davies, A.Karageorghis and T.N.Phillips, Spectral Galerkin methods for the primary two-point boundary value problem in modelling viscoelastic flows, International Journal for Numerical Methods in Engineering, vol. 26(1988), pp. 647-662.

[2] H.N.Caglar, S.H.Caglar and E.H.Twizell, The numerical solution of fifth order boundary value problems with sixth degree B-spline functions, Applied Mathematics Letters, vol. 12(1999), pp. 25-30.
[3] R.P. Agarwal, 1986, Boundary Value Problems for Higher Order Differential Equations, World Scientific, Singapore.

[4] Abdul-Majid Wazwaz, The numerical solution of fifth order boundary value problems by the Decompostion method, Journal of Computational and Applied Mathematics, vol. 136(2001), pp. 259-270.

[5] Shahidi S. Siddiqi, Ghazala Akram and Arfa Elahi, Quartic spline solution of linear fifth order boundary value problems, Applied Mathematics and Computation, vol. 196(2008), pp. 214-220.

[6] J.Rashidinia, R.Jalilian and K.Farajeyam, Spline approximate solution of fifth order boundary value problem, Applied Mathematics and Computation, vol. 192(2007), pp. 107-112.

[7] Muhammad Aslam Noor and Syed Tauseef Mohyud-Din, An efficient algorithm for solving fifth-order boundary value problems, Mathematical and Computer Modelling, vol. 45(2007), pp. 954-964.

[8] Hikmet Caglar and Nazan Caglar, Solution of fifth-order boundary value problems by using Local polynomial regression, Applied Mathematics and Computation, vol. 186(2007), pp. 952-956.

[9] Mohamed El-Gamel, Sinc and the numerical solution of fifth order boundary value problems, Applied Mathematics and Computation, vol. 187(2007), pp. 1417-1433.

[10] Muhammed I. Syam and Basem S.Ahili, Numerical solution of singularly perturbed fifth order two point boundary value problem, Applied mathematics and Computation vol. 170(2005), pp. 1085-1094.

[11] Zhao-Chunwu, Approximate analytical solutions of fifth order boundary value problems by the Variational iteration method, Computer and Mathematics with Applications, vol. 58(2009), pp. 2514-2517.

[12] A.Lamnii, H.Mraoui, D.Sbibih and A.Tijini, Sextic spline solution of fifth order boundary value problems, Mathematics and Computers Simulation, vol. 77(2008), pp. 237-246.

[13] K.N.S.Kasi Viswanadham and Sreenivasulu Ballem, Numerical solution of fifth-order boundary value problems by Galerkin method with quartic B-splines, International Journal of Computers Applications, vol. 77(2013) pp. 7-12.

[14] K.N.S.Kasi Viswanadham and S.M.Reddy, Numerical solution of fifth order boundary value problems by Petrov-Galerkin Method with quartic B-splines as basis functions and Sextic B-Splines as weight functions, International Journal of Engineering science and Innovative Technology, vol.4(1)(2015), pp.161-170.

[15] R.E.Bellman and R.E. Kalaba, 1965, Quasilinearzation and Nonlinear Boundary Value Problems, American Elsevier, New York.

[16] L.Bers, F.John and M.Schecheter, 1964, Partial Differential Equations, John Wiley Inter science, New York.

[17] J.L.Lions and E.Magenes, 1972, Non-Homogeneous Boundary Value Problem and Applications. SpringerVerlag, Berlin. 
International Journal of Computer Applications (0975 - 8887)

Volume 161 - No 10, March 2017

[18] A.R.Mitchel and R.wait, 1997, The Finite Element Method in Partial Differential Equations, John Wiley and Sons, London.

[19] P.M. Prenter, 1989, Splines and Variational Methods, John-Wiley and Sons, New York.
[20] Carl de-Boor, 1978, A Pratical Guide to Splines, Springer-Verlag.

[21] I.J. Schoenberg, 1966, On Spline Functions, MRC Report 625, University of Wisconsin. 